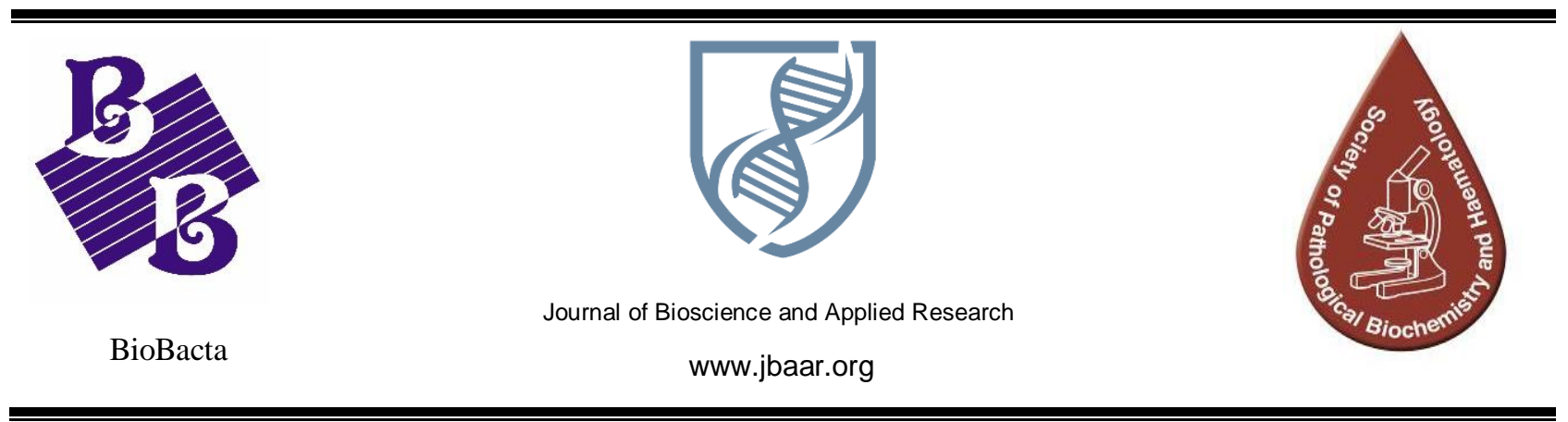

\title{
Diagnostic role of collagen III in the diagnosis of breast cancer in Egyptian women
}

\author{
Abdelfattah M. Attallah ${ }^{1 *}$, Mohamed El-Far ${ }^{2}$, Esraa A. El Sayes ${ }^{1}$, Mohamed M. Omran ${ }^{3}$ \\ ${ }^{1}$ Research \& Development Department, Biotechnology Research Center, New Damietta, Egypt \\ ${ }^{2}$ Chemistry Department, Faculty of Science, Mansoura University, Mansoura, Egypt \\ ${ }^{3}$ Chemistry Department, Faculty of Science, Helwan University, Cairo, Egypt \\ "Correspondence: Abdelfattah M. Attallah, PhD \\ Biotechnology Research Center, \\ P.O. Box (14), 23 July St., Industrial Zone, New Damietta 34517, Egypt. \\ Tel: 02/ 057 / 2429059 - 2429074, E-mail: amattallah@ hotmail.com
}

Received 15/1/2020; Accepted 10/2/2020

DOI: 10.21608/jbaar.2020.115764

\begin{abstract}
Mammography, magnetic resonance imaging (MRI), and ultrasound are useful tools that can be used in breast cancer (BC) screening but these methods have various limitations. Thus, finding diagnostic biomarkers may be a useful alternative choice. A total of 142 females with breast diseases (40 females with benign conditions and 102 females with BC) were included in the study. In addition to age- and sex-matched 29 normal females as a negative control. The level of collagen III was detected using the ELISA technique. It was $(21.29 \pm 3.14 \mu \mathrm{g} / \mathrm{ml})$ in sera of females with $\mathrm{BC}$ higher than in benign $(13.91 \pm 2.65 \mu \mathrm{g} / \mathrm{ml})$ and in normal $(5.69 \pm 0.43 \mu \mathrm{g} / \mathrm{ml})$ females. Collagen III yielded an AUC of 0.86 to differentiate BC from normal females and an AUC of 0.62 to differentiate BC females from those with benign conditions. Moreover, AUC was risen to 0.72 to differentiate females with BC from those without $\mathrm{BC}$. While the index provided values that were significantly $(P<0.01)$ higher in $\mathrm{BC}(9.1 \pm 1.3)$ than benign (6.3 \pm 1.1$)$ and normal $(2.5 \pm 1.1)$ females. It yielded an AUC of 0.98 (sensitivity $=90.0 \%)$ to differentiate $\mathrm{BC}$ from normal females and an AUC of 0.84 to differentiate $\mathrm{BC}$ females from those with benign conditions. Moreover, AUC was risen to 0.87 to differentiate females with BC from those without BC (sensitivity $=84.0 \%$ ). In conclusion, serum collagen III concentration represents a candidate biomarker for BC. Moreover, its combination with cancer antigen 15-3 (CA 15.3) and age provided a valuable index for BC diagnosis with high accuracy.
\end{abstract}

Keywords: Breast cancer, Collagen III, Index 


\section{INTRODUCTION}

Breast cancer is one of the fatal cancers that threaten the lives of women in the world. It is the most frequent cancer among females and the most frequent cause of cancer-associated death comprising $24.2 \%$ of total cancer new cases and $15 \%$ of all cancer death (Bray et al. 2018). The incidence rate increases day by day, therefore, research ongoing on the development of a superior method for early diagnosis of $\mathrm{BC}$ is a must to overcome this serious problem and to reduce mortality (Joshi, 2019; Henriksen et al. 2019).

Breast cancer screening method including mammography, clinical breast examination, MRI, and ultrasonography provide potential outcomes in BC diagnosis, but these methods improperly utilized (Weier et al. 2015), and also have various limitations including the mammography inaccurate outcomes in case of high breast density females (Shao et al. 2013; Zhao et al. 2015), the risk of ionizing radiation accompanied its use when repeated more than one time yearly. In addition, the MRI high cost, the long holding up list in developing countries and the inaccurate result of imaging techniques (Kuhl, 2019). So, finding diagnostic biomarkers may be a useful alternative choice (Tan et al. 2018).

On the other hand, using serum biomarkers may be favorable and more comfortable for patients, easily repeated and may also provide better outcomes. However, CEA, CA15-3, CA27.29, tissue polypeptide specific antigen and tissue polypeptide antigen have been suggested as diagnostic markers for BC, they have limited sensitivity and specificity for early disease (Lumachi and Basso, 2004; Menon et al. 2015; Kazarian et al. 2017).

Interestingly, the extracellular matrix (ECM) is considered one of the most important controllers in breast cancer. ECM proteins especially collagens play a major role in regulating breast health. Additionally, they undergo various changes in composition and organization in tumor progression, invasion, and metastasis (Angel et al. 2019). Therefore, the present study aimed to detect collagen III in the serum of women with BC. Then, evaluate its ability to be used as a candidate marker and in combination with other established BC markers (CA 15.3) and age for BC diagnosis.

\section{METHODS}

\section{Sample collections and laboratory assays}

In the present study, blood samples from 142 females with breast diseases, collected from the Oncology center, Mansoura University Hospitals, Mansoura, Egypt, were classified into 40 females with benign conditions and 102 females with BC. In addition, serum samples from age- and sex-matched 29 normal females were included in the study as a negative control group. None of the women with benign conditions or the normal women had a history of any malignancy. The study was commenced after obtaining approval from the university ethical and scientific committees of the Mansoura University Hospitals, Mansoura, Egypt.

Blood samples from all subjects were collected 3 days after diagnosis and before starting any cancerspecific treatment. Complete blood count (CBC) was performed on the blood portion which previously kept in K3- ethylenediamine tetraacetic acid (EDTA-K3) anti-coagulant by using automated hematologyanalyzer. The last portion of blood was left to clot and serum was separated by centrifugation at $1,000 \times \mathrm{g}$ for 10 min. Liver and kidney function tests were all measured on fresh serum on an automated biochemistry analyzer. The level of collagen III was detected using the ELISA technique according to Attallah et al protocols (Attallah et al. 2007).

\section{Statistical analysis}

Using statistical software package "SPSS 15.0, SPSS Inc.", all statistical analyses were done. Descriptive results were expressed as mean $\pm \mathrm{SD}$. Differences in 
continuous variables were assessed using analysis of variance (ANOVA). All tests were two-tailed and statistical significance assessed at the 0.05 level. Receiver-operating characteristic (ROC) curves were used to assess and compare the diagnostic accuracy of single and combined biomarkers for BC identification. Stepwise linear regression analysis was used to develop a novel index for $\mathrm{BC}$ diagnosis. The diagnostic sensitivity and specificity values were calculated from a $2 \times 2$ contingency table.

\section{RESULTS}

\section{Patient's characteristics}

Laboratory tests including liver, kidney functions, and hematology tests were carried out on normal women and women with breast diseases. As shown in (Table 1). It was found that there was no significant difference for ALT, hemoglobin, RBCs count, HCT, platelet count, WBCs and lymphocytes count $(P>$ 0.05), but there was significant difference for AST activity $(P<0.05)$, albumin level $(P<0.001)$, level of creatinine $(P<0.01)$ and neutrophil $(P<0.01)$ among the three groups.

Table 1: Patients' characteristics

\begin{tabular}{|l|c|c|c|c|}
\hline Variables & Normal & Benign disorder & Breast cancer & P \\
& $\mathbf{n = 2 9}$ & $\mathbf{n = 4 0}$ & value \\
\hline Age (Years) & $46.2 \pm 15.6$ & $47.4 \pm 11.2$ & $50.2 \pm 11.9$ & $>0.05$ \\
\hline ALT (U/L) & $28.7 \pm 6.9$ & $33.4 \pm 9.2$ & $37.2 \pm 10.4$ & $>0.05$ \\
\hline AST (U/L) & $22.6 \pm 7.3$ & $23.7 \pm 7.6$ & $25.4 \pm 6.4$ & $<0.05$ \\
\hline Albumin (g/dL) & $4.3 \pm 0.5$ & $4.1 \pm 0.4$ & $3.8 \pm 0.7$ & $<0.001$ \\
\hline Creatinine (mg/dL) & $0.60 \pm 0.07$ & $0.61 \pm 0.16$ & $0.68 \pm 0.15$ & $<0.01$ \\
\hline Hb (g/dL) & $12.3 \pm 0.9$ & $12.4 \pm 1.3$ & $12.6 \pm 1.8$ & $>0.05$ \\
\hline RBCs (10 ${ }^{6}$ cells/mL) & $4.5 \pm 0.4$ & $4.6 \pm 0.4$ & $4.7 \pm 0.5$ & $>0.05$ \\
\hline HCT (\%) & $35.7 \pm 2.7$ & $37.1 \pm 3.7$ & $38.1 \pm 4.6$ & $>0.05$ \\
\hline WBCs (10 ${ }^{6}$ cells/mL) & $7.5 \pm 1.7$ & $8.6 \pm 3.0$ & $9.0 \pm 3.3$ & $>0.05$ \\
\hline Lymphocytes (\%) & $34.5 \pm 7.7$ & $31.8 \pm 10.8$ & $29.3 \pm 12.7$ & $>0.05$ \\
\hline Neutrophil (\%) & $53.0 \pm 11.0$ & $60.5 \pm 12.7$ & $62.8 \pm 13.8$ & $<0.01$ \\
\hline Platelets (10 ${ }^{9}$ cells/L) & $245.5 \pm 45.8$ & $239.0 \pm 66.2$ & $229.8 \pm 73.8$ & $>0.05$ \\
\hline
\end{tabular}


Determination of collagen III in women with breast diseases

The level of collagen III was quantified in serum samples of females with breast diseases and normal control using ELISA. It was found that the serum collagen III level was $(21.29 \pm 3.14 \mu \mathrm{g} / \mathrm{ml})$ in serum samples of females with BC higher than in samples of females with benign breast conditions $(13.91 \pm 2.65$ $\mu \mathrm{g} / \mathrm{ml})$. While it was $(5.69 \pm 0.43 \mu \mathrm{g} / \mathrm{ml})$ in samples of normal females (Figure 1A).

\section{Diagnostic performance of collagen III for BC identification}

Using ROC curves, we assessed the diagnostic accuracy of serum collagen III antigen to differentiate between females with BC and those normal females. It yielded an area under ROC curve of 0.86 with sensitivity $=80.4 \%$ and specificity $=93.1 \%$ (Figure 1B). In addition, collagen III yielded an AUC of 0.62 and $P<0.05$ to differentiate $\mathrm{BC}$ females from those with benign conditions with sensitivity $=80.4 \%$ and specificity $=60.0 \%$. Moreover, AUC was risen to 0.72 $(P<0.05)$ to differentiate females with BC from those without $\mathrm{BC}$ with sensitivity $=80.4 \%$ and specificity $=$ $73.9 \%$ (Figures 1C-D).

\section{Development and diagnostic performance of $B C$} diagnostic index

The AUC was calculated for providing the accuracy of candidate markers for BC identification. The AUC of these candidate markers is in the order collagen III= $0.86>$ CA15.3 $=0.81>$ age $=0.69$. Then we developed a novel index for BC identification, it can be represented as Index $=0.02 \times$ CA15.3 $+0.09 \times$ age $+0.07 \times$ collagen +3.2 as consequence, the diagnostic index provided values that were significantly $(P<0.01)$ higher in BC $(9.1 \pm 1.3)$ than benign $(6.3 \pm 1.1)$ and normal $(2.5 \pm 1.1)$ women (Figure 2A).

Using ROC curves, we assessed the diagnostic accuracy of the index to differentiate between females with $\mathrm{BC}$ and those normal females. It yielded an area under ROC curve of 0.98 with sensitivity $=90.0 \%$ and specificity $=96.0 \%$ (Figure $2 \mathrm{~B}$ ). In addition, the index yielded an AUC of 0.84 to differentiate $\mathrm{BC}$ females from those with benign conditions with sensitivity = $86.0 \%$ and specificity $=79.0 \%$, Figure 2C. Moreover, AUC was risen to 0.87 to differentiate females with $\mathrm{BC}$ from those without $\mathrm{BC}$ with sensitivity $=84.0 \%$ and specificity $=80.0 \%$ (Figure 2D). 

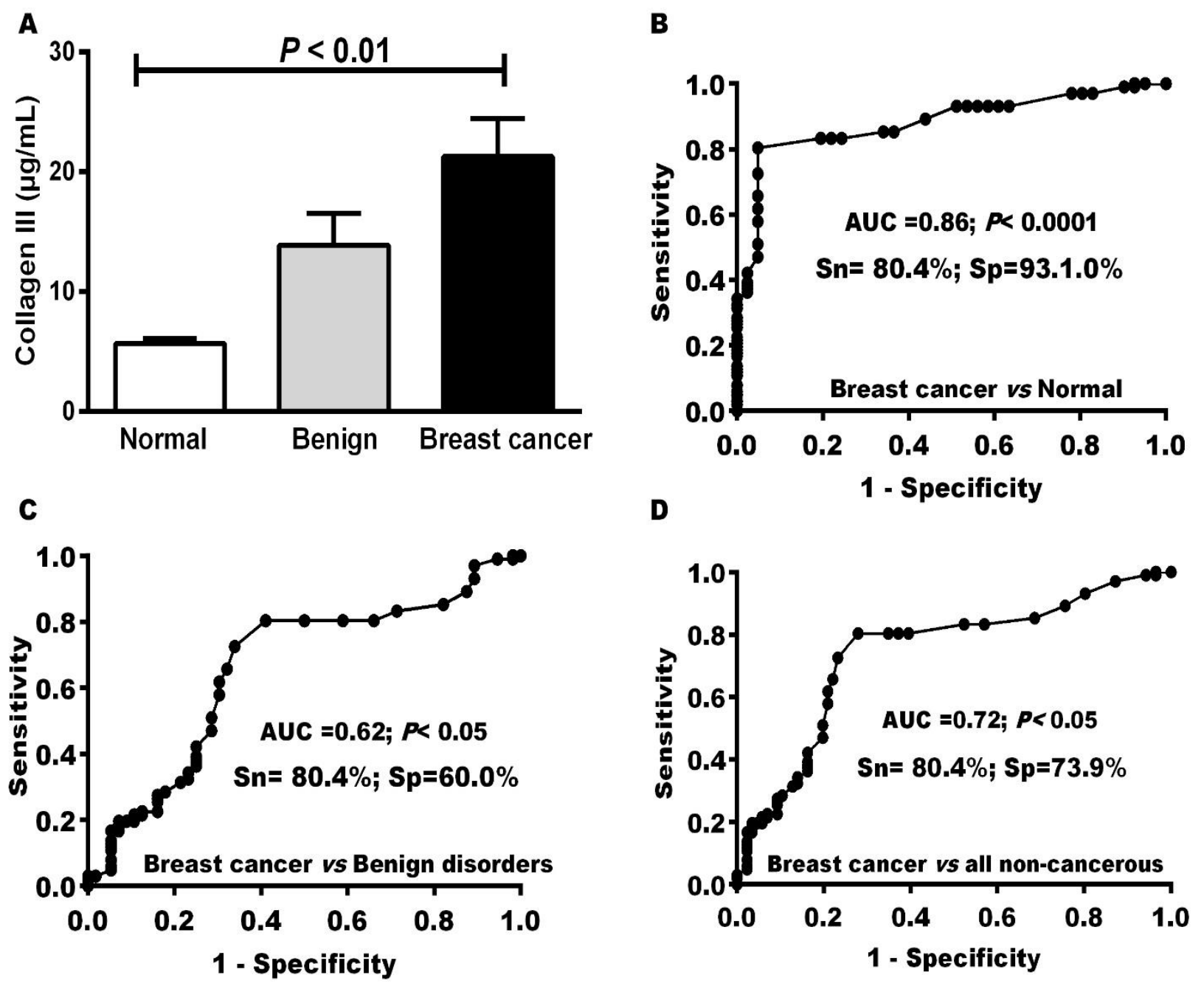

Figure 1: Diagnostic performance and distribution of collagen III. A: collagen III level among three group of subjects. Receiver operating characteristic curve of collagen III for distinguishing between breast cancer women and B: normal control, $\mathrm{C}$ : women with benign disorders and $\mathrm{D}$ : all non-cancerous women. 


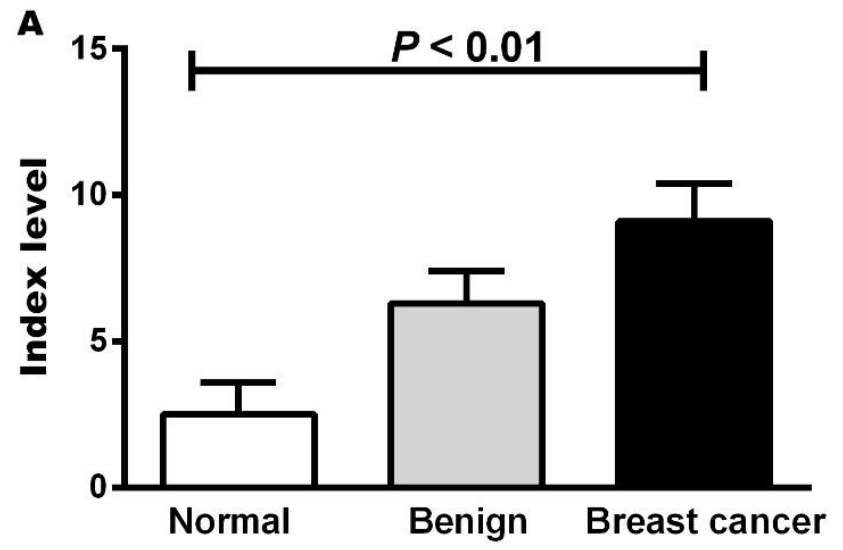

$\mathbf{B}$
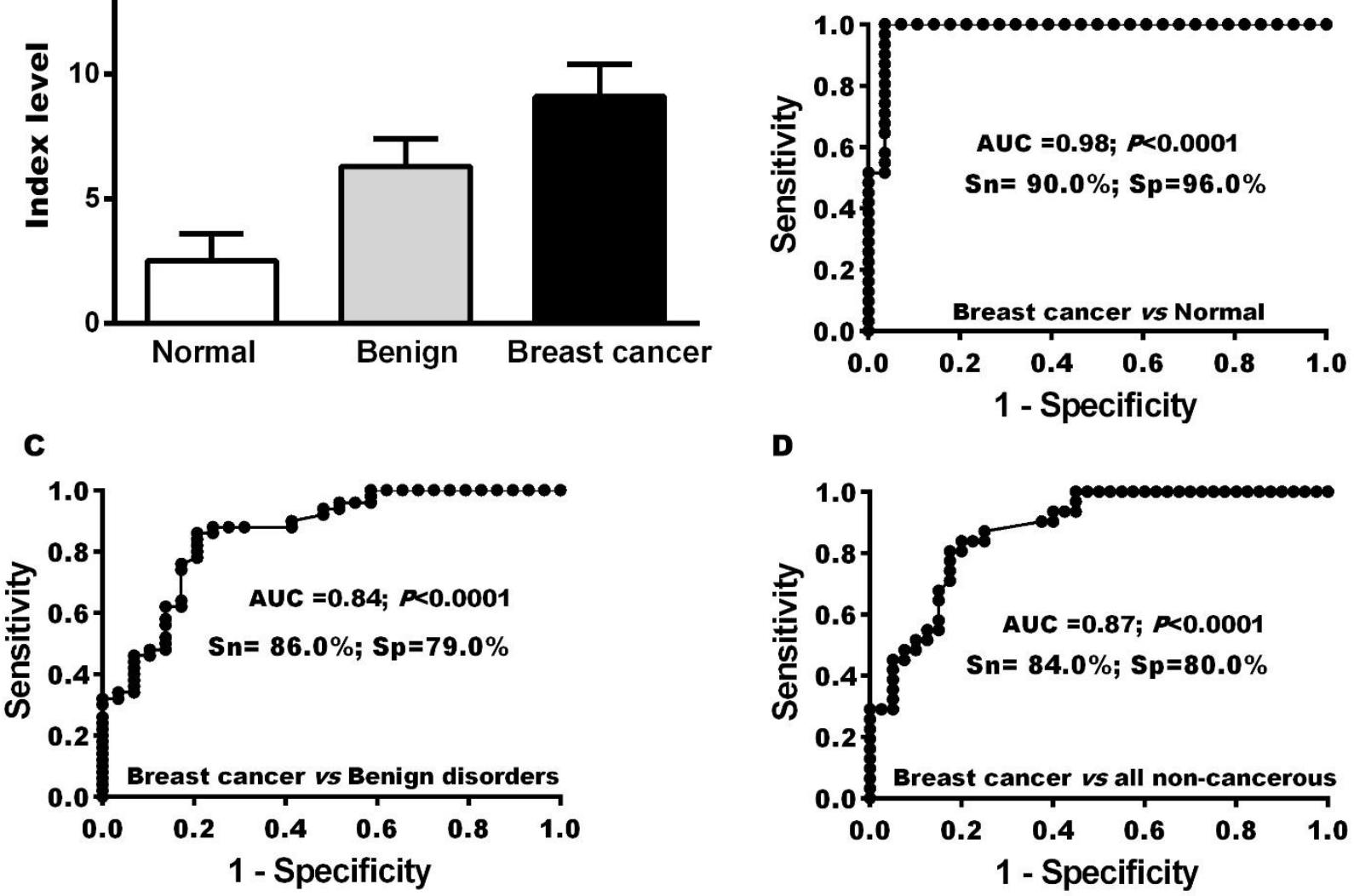

D

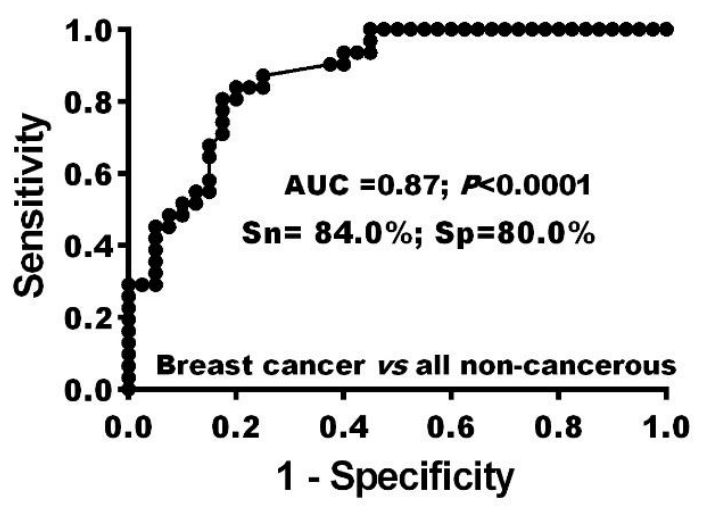

Figure 2: Diagnostic performance and distribution of Index. A: level of Index in the three group of subjects. Receiver operating characteristic curve of Index for distinguishing between breast cancer women and B: normal control, $\mathrm{C}$ : women with benign disorders and D: all non-cancerous women.

Index $=0.02 \times$ CA15.3+0.09 $\times$ age $+0.07 \times$ collagen +3.2.

\section{DISCUSSION}

Breast cancer is commonly related to extraordinary modifications of ECM structure, function, and composition (Xiong and $\mathrm{Xu}, \mathbf{2 0 1 6}$ ). It is also associated with the increased breast density and the over deposition of collagens such as collagen I, II, and III which involved in tumor progression and invasion as it involved in altering tumor stroma structure through series of enzymatic reaction that results in increasing its thickness, stiffness, crosslinking, degradation, redeposition and accordingly altered ECM remodeling and facilitate tumor progression (Fang et al. 2014). Collagen III is one of the main fibrillar structural proteins of ECM which comprises $10-15 \%$ of skin collagen (Riekki et al. 2004; Mazurek et al. 2018). Mutation in this collagen involved in several malignancies and it can be used as a biomarker (Nielsen and Karsdal, 2016). 
In this study, we found that there was a high significant increase $(P<0.01)$ in serum collagen III levels in $\mathrm{BC}$ women than in women with a benign condition $(P<0.01)$ and normal control. These results were in agreement with Bager et al. (2015) who found that there was a high significant increase in serum level of collagen type I, III and IV in BC patients $(P<0.04$ 0.0001) compared to healthy controls. Rizwan et al. (2015) also found that there was up-regulation in the gene expression of collagen III alpha $1(P=0.00206)$. These findings are in accordance with previous reports which found elevated levels of type III collagen in the tissue of BC patients (Kauppila et al. 1998).

In breast cancer, several matrix metalloproteases expression are significantly increased that correlated with lysyl oxidase expression which increases the ECM stiffness as it has a collagen crosslinking activity and induces stromal collagen to secrete more matrix metalloproteinase which degrades collagen and promote tumor progression (Motrescu et al. 2008; Erler et al. 2009)

In this study, AUC of collagen III to differentiate women with $\mathrm{BC}$ from those normal women was 0.86 with high sensitivity and specificity (80.4\% and $93.1 \%$; respectively). Also, it yielded high sensitivity and specificity to differentiate $\mathrm{BC}$ women from those women without $\mathrm{BC}(\mathrm{AUC}=0.72$, sensitivity $=80.4 \%$ and specificity $=73.9 \%)$ with significant difference $(P$ $<0.05)$. Moreover, collagen III still had a good diagnostic performance to differentiate women with $\mathrm{BC}$ from those women with benign conditions (AUC $=0.62$, yield sensitivity $=80.4 \%$ and specificity $=60.0 \%)$ with significant difference $(P<0.05)$.

While the AUC of the index to differentiate women with $\mathrm{BC}$ from those normal women was 0.98 with high sensitivity and specificity $(90.0 \%$ and $96.0 \%$; respectively). Also, it yielded high sensitivity and specificity to differentiate $\mathrm{BC}$ women from those women without $\mathrm{BC}(\mathrm{AUC}=0.87$, sensitivity $=84.0 \%$ and specificity $=80.0 \%)$ with significant difference $(P$ $<0.0001)$. Moreover, the index still had a good diagnostic performance to differentiate women with $\mathrm{BC}$ from those women with benign conditions (AUC $=0.84$, yield sensitivity $=86.0 \%$ and specificity $=79.0 \%)$ with significant difference $(P<0.0001)$.

Collagen III provided a diagnostic performance superior to other BC markers, such as CEA, CA 15.3, CA125, CA 19-9, CA 27.29, total and free prostatespecific antigen which they yielded sensitivities (18\%, 31.9\%, 29.8\%, 14\% (Zaleski et al. 2018), 62\% (De Paterna et al. 1995), 57\% and 36\%; respectively ) (Black et al. 2000). Furthermore, it is superior to CEA, CA 15.3, CA125, CA 19-9, total and free prostatespecific antigen in distinguishing women with $\mathrm{BC}$ from women with benign conditions which they yielded sensitivities (20\%, 23.4\%, 19.1\%, 6\% (Zaleski et al. 2018), $57 \%$ and $36 \%$; respectively). However, free the prostate specific-antigen is more specific in distinguishing women with $\mathrm{BC}$ from women with benign conditions than total prostate specific-antigen and collagen III Where the specificities were $72 \%$, 25\%, and 60\%; respectively (Black et al. 2000).

The ROC curve also showed diagnostic performance of the epidermal growth factor to differentiate patients with $\mathrm{BC}$ from those without $\mathrm{BC}$ superior to collagen III with $\mathrm{AUC}=0.89$ and sensitivity $94.0 \%$ but collagen III is more specific than the epidermal growth factor which they yielded specificities (73.9\% and 70.6\%, respectively) (Kim et al. 2009).

Concerning the diagnostic performance of the index, it provided AUC superior to the combination of CEA with CA15-3 and the combination of CEA or CA15-3 with miR-34a to differentiate women with $\mathrm{BC}$ from those normal women which they yielded AUC of 0.74 , 0.84 and 0.80 ; respectively and the sensitivities were (38.5, 34.1 and 56.1; respectively). Moreover, it is superior to the combination of CEA with miR-34a and 
the combination of CA15-3 with miR-34a to differentiate women with $\mathrm{BC}$ from those women with benign conditions which they yielded sensitivities (31.8 and 53.7; respectively) (Zaleski et al. 2018).

Furthermore, the index provided AUC superior to the combination of collagen III, nuclear matrix protein-52 and matrix metalloproteinase-1 to differentiate women with $\mathrm{BC}$ from those with benign conditions which they yielded AUC of 0.73 and the sensitivity of $78 \%$. However, the AUC of the index is slightly lower than the AUC of this combination (AUC, 0.99) in distinguishing women with $\mathrm{BC}$ from normal women (Attallah et al. 2019).

Moreover, the index diagnostic performance is lower than the diagnostic performance of the combination of vascular endothelial growth factor, tissue inhibitors of metalloproteinase-2 and CA 15-3 which they yielded a sensitivity of $93 \%$ (Lawicki et al. 2017).

\section{CONCLUSION}

In conclusion, serum collagen III concentration represents a candidate biomarker for $\mathrm{BC}$, which was detected using ELISA, in higher concentration in BC females compared to normal and females with benign breast conditions. It had also a potential role in detecting $\mathrm{BC}$ with high sensitivity and specificity. Moreover, the combination of collagen III with other established BC marker (CA 15.3) and age provided a valuable index for $\mathrm{BC}$ diagnosis with high accuracy.

\section{REFERENCES}

Angel, P.M., Schwamborn, K., Comte-Walters, S., Clift, C.L., Ball, L.E., Mehta, A.S., and Drake, R.R. (2019). Extracellular matrix imaging of breast tissue pathologies by MALDI-imaging mass spectrometry. Proteomics Clin Appl 13, 1700152.

Attallah, A.M., Mosa, T.E., Omran, M.M., Abo Zeid, M.M., El-Dosoky, I., and Shaker, Y.M. (2007). Immunodetection of collagen types I, II, III, and IV for differentiation of liver fibrosis stages in patients with chronic HCV. J Immunoassay Immunochem 28, 155168.

Attallah, A.M., El-Far, M., Fatah, M.E., Omran, M.M., Abdelrazek, M.A., Abdelhameed, G.E., Attallah, K.A., Ahmed, N.A., El-sayes, E.A., Khedr, F.M., and El-Dosoky, I. (2019). Combining nuclear matrix protein-52, collagen III and matrix metalloproteinase-1 for more effective breast cancer early detection. J Biosci Appl Res 5, 212 -221.

Bager, C.L., Willumsena, N., Leeming, D.G., Smith, V., Karsdal, M.K., Dornan, D., and Bay-Jensen, A.C. (2015). Collagen degradation products measured in serum can separate ovarian and breast cancer patients from healthy controls: a preliminary study. Cancer Biomarker 15, 783-788.

Black, M.H., Giai, M., Ponzone, R., Sismondi, P., Yu, H., and Diamandis, E.P. (2000). Serum total and free prostate-specific antigen for breast cancer diagnosis in women. Clin Cancer Res 6, 467-473.

Bray, F., Ferlay, J., Soerjomataram, I., Siegel, R.L., Torre, L.A., and Jemal, A. (2018). Global cancer statistics 2018: Globocan estimates of incidence and mortality worldwide for 36 cancers in 185 countries. CA Cancer J Clin 68, 394-424.

De Paterna, L.R., Arnaiz, F., Estenoz, J., Ortuno, B., and Lanzos, E. (1995). Study of serum tumor markers CEA, CA 15.3 and CA 27.29 as diagnostic parameters in patients with breast carcinoma. Int J Biol Markers $10,24-29$.

Erler, J.T., Bennewith, K.L., Cox, T.R., Lang, G., Bird, D., Koong, A., Le, Q.T., and Giaccia, A.J. (2009). Hypoxia-induced lysyl oxidase is a critical mediator of bone marrow cell recruitment to form the premetastatic niche. Cancer Cell 15, 35-44. 
Fang, M., Yuan, J., Peng, C., and Li, Y. (2014). Collagen as a double-edged sword in tumor progression. Tumour Biol 35, 2871-2882.

Henriksen, E.L., Carlsen, J.F., Vejborg, I.M., Nielsen, M.B., and Lauridsen, C.A. (2019). The efficacy of using computer-aided detection (CAD) for detection of breast cancer in mammography screening: a systematic review. Acta Radiol 60, 13-18.

Joshi, D. (2019). Breast cancer treatment: survival facts and associated side effects. HJHS 26, 34-40.

Kauppila, S., Stenbäck, F., Risteli, J., Jukkola, A., and Risteli, L. (1998). Aberrant type I and type III collagen gene expression in human breast cancer in vivo. J Pathol 186, 262-268.

Kazarian, A., Blyuss, O., Metodieva, G., GentryMaharaj, A., Ryan, A., Kiseleva, E.M., Prytomanova, O.M., Jacobs, I.J., Widschwendter, M., Menon, U., and Timms, J.F. (2017). Testing breast cancer serum biomarkers for early detection and prognosis in pre-diagnosis samples. Br J Cancer 116, 501-508.

Kim, B.K., Lee, J.W., Park, P.J., Shin, Y.S., Lee, W.Y., Lee, K.A., Ye, S., Hyun, H., Kang, K.N., Yeo, D., and Kim, Y. (2009). The multiplex bead array approach to identifying serum biomarkers associated with breast cancer. Breast Cancer Res 11, 22-33.

Kuhl, C.K. (2019). Abbreviated magnetic resonance imaging (MRI) for breast cancer screening: rationale, concept, and transfer to clinical practice. Annu Rev Med 70, 501-519.

Lawicki, S., Zajkowska, M., Glażewska, E.K., Będkowska, G.E., and Szmitkowski, M. (2017). Plasma levels and diagnostic utility of VEGF, MMP-2 and TIMP-2 in the diagnostics of breast cancer patients. Biomarkers 22, 157-164.
Lumachi, F. and Basso, S.M. (2004). Serum tumor markers in patients with breast cancer. Expert Rev Anticancer Ther 4, 921-931.

Mazurek, K., Siemianowicz, K., Likus, W., Pierzchała, E., Kwiatkowski, R., and Markowski, J. (2018). Collagen type III metabolism evaluation in patients with malignant head and neck cancer treated with radiotherapy. Biomed Res Int 15, 9546-9565.

Menon, U., Ryan, A., Kalsi, J., Gentry-Maharaj, A., Dawnay, A., Habib, M., Apostolidou, S., Singh, N., Benjamin, E., and Burnell, M., Davies, S. (2015). Risk algorithm using serial biomarker measurements doubles the number of screen-detected cancers compared with a single-threshold rule in the United Kingdom collaborative trial of ovarian cancer screening. J Clin Oncol 33, 2062-2071.

Motrescu, E.R., Blaise, S., Etique, N., Messaddeq, N., Chenard, M.P., Stoll, I., Tomasetto, C., and Rio, M.C. (2008). Matrix metalloproteinase11/stromelysin-3 exhibits collagenolytic function against collagen VI under normal and malignant conditions. Oncogene 27, 6347-6355.

Nielsen, M.J. and Karsdal, M.A. (2016). Type III collagen. In: Karsdal, M.A., Leeming, D.J., Henriksen, K., and Bay-Jensen, A., edts, Biochemistry of collagens, laminins and elastin: structure, function and biomarkers. 1st ed. Academic Press, pp 21-30.

Riekki, R., Harvima, I.T., Jukkola, A., Risteli, J., and Oikarinen, A. (2004). The production of collagen and the activity of mast-cell chymase increase in human skin after irradiation therapy. Exp Dermatol 13, 364-371.

Rizwan, A., Bulte, C., Kalaichelvan, A., Cheng, M., Krishnamachary, B., Bhujwalla, Z.M., Jiang, L., and Glunde, K. (2015). Metastatic breast cancer cells in lymph nodes increase nodal collagen density. Sci Rep 5, 10002. 
Shao, H., Li, B., Zhang, X., Xiong, Z., Liu, Y., and

Tang, G. (2013). Comparison of the diagnostic efficiency for breast cancer in Chinese women using mammography, ultrasound, MRI, and different combinations of these imaging modalities. J Xray Sci Technol 21, 283-292.

Tan, G., Chu, C., Gui, X., Li, J., and Chen, Q.

(2018). The prognostic value of circulating cell-free DNA in breast cancer: a meta-analysis. Medicine 97, 0197- 0197.

Wang, G., Qin, Y., Zhang, J., Zhao, J., Liang, Y.A., Zhang, Z., Qin, M., and Sun, Y. (2014). Nipple discharge of CA15-3, CA125, CEA and TSGF as a new biomarker panel for breast cancer. Int J Mol Sci 15, 9546-9565.

Weier, R.C., Reisinger, S.A., and Paskett, E.D. (2015). Breast cancer screening. In Holland, J.C., Breitbart, W.S., Jacobsen, P.B., Loscalzo, M.J., McCorkle, R., and Butow, P.N., edts, PsychoOncology. $3^{\text {rd }}$ ed. Oxford University Press. New York, pp58-63.

Xiong, G.F., and Xu, R. (2016). Function of cancer cell-derived extracellular matrix in tumor progression. J Cancer Metastasis Treat 2, 358-364.

Zaleski, M., Kobilay, M., Schroeder, L., Debald, M., Semaan, A., Hettwer, K., Uhlig, S., Kuhn, W., Hartmann, G., and Holdenrieder, S. (2018). Improved sensitivity for detection of breast cancer by combination of miR-34a and tumor markers CA 15-3 or CEA. Oncotarget 9, 22523-22536.

Zhao, H., Zou, L., Geng, X., and Zheng, S. (2015). Limitations of mammography in the diagnosis of breast diseases compared with ultrasonography: a singlecenter retrospective analysis of 274 cases. Eur J Med Res 20, 49-55. 